\title{
Internacionalización de la educación superior y \\ TIC. La mirada de los organismos internacionales
}

\author{
Manzanilla-Granados, Héctor Manuel \\ Escuela Superior de Cómputo del Instituto Politécnico Nacional, Ciudad de México \\ hmanzanilla@ipn.mx
}

Navarrete-Cazales, Zaira

Facultad de Filosofía y Letras-Universidad Nacional Autónoma de México, Ciudad de México znavarretec@gmail.com

\section{Resumen}

Algunos organismos internacionales como la Organización de las Naciones Unidas para la Educación, la Ciencia y la Cultura (UNESCO), la Comisión Económica para América Latina y el Caribe (CEPAL), el Banco Interamericano de Desarrollo (BID), la Organización de Estados Iberoamericanos para la Educación, la Ciencia y la Cultura (OEI), entre otros, han realizado recomendaciones para que todos los países se inserten en «un mundo globalizado» y una de las herramientas que permite el acceso a la globalización son las TIC. Independientemente de los retos que organismos como la UNESCO plantean a la educación superior en el mundo actual, no es fácil asumirlos sin tener en cuenta que la dimensión de la educación superior tiene varios indicadores que nos permiten reconocerla como compleja, multifacética, diversa, cambiante y desafiante.

La internacionalización de la educación superior implica nuevas formas de cooperación. Las agencias internacionales han definido ya toda una agenda de cambios para las universidades. Ahora se pretende conformar una universidad que responda fundamentalmente a las demandas expresadas mediante la circulación de recursos y el mercado, es decir, a las demandas de la globalización. El objetivo de este escrito es presentar una revisión teórica-documental, en la que analizamos documentos bases emitidos por Organismos internacionales sobre el tema de la internacionalización de la educación superior.

Se concluye que cada vez más la educación superior, a nivel mundial, tiene que responder a tendencias macro que la están afectando como el movimiento hacia una sociedad y economía del conocimiento; el desarrollo en tecnología informática y comunicaciones; la mayor movilidad de la gente, capital, ideas, conocimiento y tecnología; el mayor énfasis en la economía de mercado; los cambios en la gobernabilidad, desde lo nacional a regional e internacional; así como también la importancia cada vez mayor de un aprendizaje permanente para toda la vida.

Palabras clave: Internacionalización, educación superior, TIC, organismos internacionales.

\section{INTRODUCCIÓN}

Generalmente el término internacionalización se utiliza para referirse a las relaciones entre las naciones, al nuevo impulso que han tomado las relaciones internacionales, el comercio internacional, los tratados y las alianzas entre países. Para la internacionalización las unidades de análisis continúan siendo los estados nacionales (Navarro y Navarrete, 2016).

La internacionalización de la educación superior se remonta a los inicios mismos de las primeras universidades en el mundo, por ejemplo, Bolonia y Salamanca. Tanto si ésta es entendida como movilidad académica de estudiantes y docentes, o si la entendemos como programas académicos internacionales. Universidades «internacionales ayer en el ámbito de la Cristiandad con alumnos que peregrinaban de un lugar a otro formando las "naciones" estudiantiles y con profesores que ya al inicio de esta experiencia institucional-cultural conocieron el ius ubique docendi, el derecho de enseñar en cualquier parte del mundo cristiano, ellas prolongan dichas raíces en un mundo secularizado, global y posmoderno» (Brunner, 2008: 122).

El término «internacionalización» emerge a partir del fenómeno de la globalización (también denominada mundialización) y se adjudica al terreno de la economía capitalista o del neocapitalismo con el objetivo de interrelacionarse con otros países, principalmente de mantener interrelaciones 
con otras economías del mundo con el objetivo de mejorar la propia economía a través del intercambio y desarrollo científico y tecnológico, lo cual demanda una «mejor» formación de científicos y tecnólogos(Brunner, 2010). Cabe señalar que los términos globalización e internacionalización son complementarios en tanto que la globalización corresponde a las fuerzas económicas, políticas y sociales que empujan la educación superior del siglo XXI hacia una mayor implicación en la escena internacional. Estos factores incluyen la creciente integración de la investigación, el uso del inglés como lingua franca para la comunicación científica y, de algún modo, para la educación superior a nivel mundial, la importancia cada vez mayor de un mercado laboral internacional para los académicos y científicos y, más particularmente, todos los aspectos relacionados con la tecnología de la información. La tecnología de la información facilita la comunicación, permite mejorar el almacenamiento, la selección y la difusión del saber a todos los niveles y vuelve posible la oferta de programas académicos de todo tipo por medio de lo que se ha llamado el aprendizaje mediante redes electrónicas o e-learning (Altbach y Knight, 2006).

En este escrito presentaremos una sucinta revisión teórica-documental, en la que analizamos documentos bases emitidos por Organismos internacionales sobre el tema de la internacionalización de la educación superior.

\section{INTERNACIONALIZACIÓN DE LA EDUCACIÓN SUPERIOR. NOTAS CONCEPTUALES}

El término internacionalización se ha recuperado en el terreno educativo, específicamente en el de la educación superior, con el objetivo de formar cada vez mejor a los nuevos profesionistas principalmente por medio del intercambio estudiantil (Navarrete y Málaga, 2014).

En la actualidad, cuando se habla de internacionalización de la educación superior se evoca primordialmente el Proceso de Bolonia, el cual tuvo sus orígenes a mediados de los años 80 en concordancia con la Unión Europea, cuyo objetivo principal fue aumentar la movilidad académica al interior de la región. Sin embargo, hoy en día se resalta el mayor énfasis que se la ha dado a la internacionalización en casa como una corriente paralela a la movilidad académica. Lo anterior no significa que se excluyen totalmente, pero sí existen elementos diferenciadores marcados (Moreno, 2016).

La internacionalización de la educación superior se ha acelerado primordialmente por la globalización por lo tanto es necesario adoptar transformaciones necesarias en la educación superior que se adapten a los cambios que se han dado en esta época, la internacionalización representa un campo de correlación disciplinar y promueve el interés de especialistas de distintas profesiones, que aunque pocas veces actúan interdisciplinariamente hacen que ciertas valoraciones ideológicas sean más claras en algunos países que en otros (Didou, 2014).

En la práctica social, la internacionalización de la educación -superior- se propone como un modelo a seguir por todas las universidades que quieran estar a la «vanguardia» del conocimiento. Es decir, como un modelo de identificación que las universidades deberán imitar (Navarrete, 2010). No obstante, la internacionalización de la educación superior se ha entendido de diferentes maneras por los diversos actores participantes en este proceso. Los proyectos de desarrollo internacionales han sido percibidos tradicionalmente como parte de la internacionalización y más recientemente, el incremento del énfasis en el comercio de la educación superior, también está siendo visto como internacionalización. Así, la internacionalización es interpretada y utilizada de forma diferente en los países alrededor del mundo (Knight, 2010).

\section{ORGANISMOS INTERNACIONALES: INTERNACIONALIZACIÓN DE LA EDUCACIÓN SUPERIOR Y TIC}

Algunos organismos internacionales como la Organización de las Naciones Unidas para la Educación, la Ciencia y la Cultura (UNESCO), la Comisión Económica para América Latina y el Caribe (CEPAL), el Banco Interamericano de Desarrollo (BID), la Organización de Estados Iberoamericanos para la Educación, la Ciencia y la Cultura (OEI), entre otros, han realizado recomendaciones para que todos los países se inserten en "un mundo globalizado» y una de las herramientas que permite el acceso a la globalización son las TIC. Independientemente de los retos que organismos como la UNESCO 
plantean a la educación superior en el mundo actual, no es fácil asumirlos sin tener en cuenta que la dimensión de la educación superior tiene varios indicadores que nos permiten reconocerla como compleja, multifacética, diversa, cambiante y desafiante. Cada vez más la educación superior, a nivel mundial, tiene que responder a tendencias macro que la están afectando como el movimiento hacia una sociedad y economía del conocimiento; el desarrollo en tecnología informática y comunicaciones; la mayor movilidad de la gente, capital, ideas, conocimiento y tecnología; el mayor énfasis en la economía de mercado; los cambios en la gobernabilidad, desde lo nacional a regional e internacional; la privatización de la educación, así como también la importancia cada vez mayor de un aprendizaje permanente para toda la vida (Castellanos y Luna, 2009).

En tal sentido, por medio de una revisión teórica-documental, daremos cuenta de algunas recomendaciones internacionales y cómo se han resignificado éstas en el ámbito de la educación superior mexicana, específicamente sobre la oferta de educación a distancia que hace uso de las TIC como una herramienta posibilitadora de inclusión y cohesión, al desaparecer barreras geográficas, todo ello en el marco de la internacionalización de la educación superior. Vivimos en un mundo globalizado en el que cada día hacemos más uso de las TIC y por ello algunos teóricos han denominado a esta nueva época como: Aldea global (McLuhan, 1986), Era Tecnotrónica (Zbigniew, 1973), Sociedad Postindustrial (Bell, 2006), Sociedad de la Información (Castell, 2002), Sociedad del Conocimiento (Drucker, 1994), entre otras. De acuerdo con Kofi Annan, todas estas denominaciones tienen en común la idea de que las Tecnologías de la Información y de la Comunicación pueden mejorar la vida de los seres humanos en cualquier lugar del mundo (Annan, 2003). Se disponen de herramientas para llegar a los Objetivos de Desarrollo del Milenio, de instrumentos que harán avanzar la causa de la libertad y la democracia, y de los medios necesarios para propagar los conocimientos y facilitar la comprensión mutua (Paliwala, 2006).

Se ha dicho que la educación continua y de adultos, la formación en «otras disciplinas» y el aprendizaje a lo largo de la vida, la enseñanza a distancia y «otros servicios especiales», pueden ser una contribución clave para la ocupabilidad y ayudar a las personas a aprovechar las nuevas posibilidades que ofrecen las TIC para los empleos tradicionales, el trabajo por cuenta propia y las nuevas profesiones. En este sentido, la sensibilización y la alfabetización en el ámbito de las TIC son un sustento fundamental (WSIS, 2004). Estamos presenciando los cambios acelerados y transformaciones profundas que enfrenta la sociedad y que repercuten en los procesos educativos. De esta manera, lo global y lo local interactúan para producir una «cultura global», glocal (Robertson, 1992). Las TIC son una herramienta fundamental que viene de la mano con la globalización. Uno de los pivotes de la ideología que exalta el valor clave de la educación superior tiene que ver con la idea de que el capitalismo global funciona hoy, en sus sectores más dinámicos, en torno al uso intensivo y la aplicación innovadora del conocimiento. Para medir el avance de los países hacia esta economía basada en el conocimiento, el Banco Mundial ha diseñado un Índice que evalúa cuatro factores considerados esenciales para la participación de los países en dicha economía: su régimen de incentivos económicos; capacidades de innovación; educación, y la penetración y uso de nuevas tecnologías de información y comunicación (Brunner, 2010).

En la actualidad, nada hace pensar que el ritmo de la evolución tecnológica se vea minimizado, ante tal situación es necesario que todos y cada uno de los miembros imbricados en el terreno educativo, en todas sus modalidades, se apropien de estos cambios, tal como lo señalan organismos internacionales como la UNESCO, la CEPAL, el BID y la OEI. En tal sentido, la UNESCO señala que las TIC pueden contribuir al acceso universal a la educación, la igualdad en la instrucción, el ejercicio de la enseñanza y el aprendizaje de calidad y el desarrollo profesional de los docentes, así como a la gestión dirección y administración más eficientes del sistema educativo, en tal sentido aplica una estrategia amplia e integradora en lo tocante a la promoción de las TIC en la educación. El acceso, la integración y la calidad figuran entre los principales problemas que las TIC pueden abordar. El dispositivo intersectorial de la UNESCO para el aprendizaje potenciado por las TIC aborda estos temas mediante la labor conjunta de sus tres sectores: Comunicación e Información, Educación y Ciencias (UNESCO, 2013).

La red mundial de oficinas, institutos y asociados de la UNESCO facilita a los Estados Miembros los recursos para elaborar políticas, estrategias y actividades relativas al uso de las TIC en la educación. En particular, el Instituto de la UNESCO para la Utilización de las Tecnologías de la Información en la Educación, con sede en Moscú, se especializa en el intercambio de información, la investigación y la capacitación con miras a integrar las TIC en la enseñanza, mientras que la Oficina de la UNESCO en Bangkok mantiene una intensa participación en lo tocante al uso de las TIC en la educación, en la región de Asia y el Pacífico (UNESCO, 2013). 
Por otra parte, el BID ha reconocido desde hace mucho la importancia de la ciencia y tecnología y ha hecho contribuciones importantes para fortalecer las capacidades nacionales desde que comenzó a otorgar préstamos en 1962. EI BID tiene entre sus objetivos apoyar la función de las instituciones de tecnología en relación con las pequeñas y medianas empresas, que tradicionalmente no han utilizado de manera adecuada estos servicios. Dar apoyo financiero para crear la infraestructura (instalaciones y equipo), formar personal, emprender las actividades iniciales (los gastos operativos iníciales) y proporcionar donaciones de contrapartida para los servicios pagados por las empresas. El nivel de los subsidios dependerá de la función o la actividad (Fonseca, 2001).

La CEPAL desde sus inicios ha considerado la educación como un eslabón que contribuye a conciliar el crecimiento, la equidad y la participación en la sociedad. La educación juega un papel central en el crecimiento de las economías, ya que es una inversión con alta tasa de retorno y es un factor que dinamiza la creación de valor. Individuos más educados aumentan sus capacidades para contribuir más y de modo más diversificado y eficiente al desarrollo productivo de un país. Por otro lado, la educación es uno de los principales campos de reducción de desigualdades a futuro y una de las vías privilegiadas para superar la pobreza. Lo anterior se debe a los círculos virtuosos entre mayor educación, movilidad sociocupacional y mejores ingresos. Adicionalmente, en el umbral del siglo XXI marcado por conflictos culturales y debilitamiento de las certezas, la educación se vuelve una herramienta para repensar críticamente la realidad y aportar con proyectos e ideas que promuevan una visión multicultural (CEPAL-OEI, 2010).

Tanto la UNESCO como la CEPAL, han destacado que para alcanzar las metas establecidas es necesario modernizar la gestión de la educación, profesionalizar y dar protagonismo a los educadores, otorgar un papel más relevante a la comunidad educativa local y una acción más estratégica a la administración central. Pero además, es necesario incorporar las TIC al proceso de enseñanza y aprendizaje, orientar el currículo hacia la adquisición de las competencias básicas, formar ciudadanos activos y responsables, asegurar la conexión de la educación con los anhelos de los jóvenes y lograr su participación activa en su propia formación (CEPAL-OEI, 2010).

En este sentido, es indispensable que todos las personas inmiscuidas con la educación (directivos, administrativos, profesores y alumnos) incorporen en su actividad herramientas tecnológicas, principalmente la integración de la computadora y la World Wide Web en los proceso de su competencia o de su cargo (de dirección, organización, gestión, administración, enseñanza, aprendizaje), puesto que la incorporación de estos medios y la adecuada utilización didáctica de los mismos suponen mejoras metodológicas para el desempeño de dichas actividades. Anteriormente se creía que sólo los profesores y alumnos debían hacer uso de las TIC, actualmente se hace necesario el uso por parte de la mayoría de los integrantes de una comunidad educativa, máxime si se trata de una institución de educación superior.

\section{CONCLUSIONES}

El entorno mundial y la naturaleza transfronteriza del conocimiento exigen a las naciones, en el futuro inmediato, impulsar políticas, estrategias y programas más eficientes para la internacionalización de la educación superior (Valls, 2015).

La globalización ha impuesto nuevos retos a las universidades, se están cambiando las tradicionales formas de cooperación internacional e interuniversitaria. La internacionalización de la educación superior implica nuevas formas de cooperación. Las agencias internacionales han definido ya toda una agenda de cambios para las universidades. Ahora se pretende conformar una universidad que responda fundamentalmente a las demandas expresadas mediante la circulación de recursos y el mercado, es decir, a las demandas de la globalización.

La formación-preparación en TIC augura, desde los discursos institucionales, una mejor posibilidad de vida, de recortar la brecha digital entre los países más atrasados, además de servir como motor de desarrollo económico y de ascenso en el nivel social. De tal manera que la educación a distancia supone la aplicación de nuevas tecnologías al proceso educativo. En el Foro Mundial sobre la Educación 2015 (UNESCO, 2015), se estableció como uno de sus acuerdos promover oportunidades de aprendizaje de calidad a lo largo de la vida para todos, en todos los contextos y en todos los niveles 
educativos, así como a fortalecer la ciencia, la tecnología y la innovación. Se recomendó aprovechar las TIC para reforzar los sistemas educativos, la difusión de conocimientos, el acceso a la información, el aprendizaje efectivo y de calidad, y una prestación más eficaz de servicios.

\section{REFERENCIAS BIBLIOGRÁFICAS}

Altbach, P. y Knight, J. (2006). «Visión panorámica de la internacionalización en la educación superior: motivaciones y realidades» en Perfiles Educativos, Vol. XXVIII, No. 112, pp 13-39.

Annan, K. (2003). Discurso inaugural de la primera fase de la World Summit on Information Society (WSIS), Ginebra: Organización de las Naciones Unidas.

Bell, D. (2006). El advenimiento de la sociedad post-industrial. Buenos Aires: Alianza Editorial.

Brunner, J.J. (2008). «El proceso de Bolonia en el horizonte Latinoamericano: límites y posibilidades», en Revista de Educación, Número extraordinario 2008: Tiempos de cambio universitario en Europa. pp. 119-145. Madrid. Recuperado de: http://www.revistaeducacion.mec.es/re2008/re2008.pdf [Consulta: 09.08.2016].

Brunner, J.J. (2010). «Globalización de la educación superior: crítica de su figura ideológica», en Revista Iberoamericana de Educación Superior, México, ISSUE-UNAM/Universia, vol. I, núm.2, pp. 75-83, Recuperado de http://ries.universia. net/index.php/ries/article/view/56/globalizacion [Consulta: 02.10.2016].

Castellanos, S. y Luna C. (2009). «La internacionalización y la globalización neoliberal en el contexto de la educación superior en México» en Revista Iberoamericana de educación, Organización de Estados Iberoamericanos para la Educación, la Ciencia y la Cultura (OEI), No. 49/7, pp. 1-10.

Castells, M. (2002). La Era de la Información. Vol. I: La Sociedad Red. México: Siglo XXI Editores.

CEPAL-OEI, (2010) «2021 Metas Educativas. La educación que queremos para la generación de los bicentenarios». España: OEl. Recuperado de http://www.oei.es/metas2021.pdf [Consulta: 22.07.2016].

Didou, S. (2014) «Introducción. La Internacionalización de la Educación Superior y la Ciencia en América Latina: Un balance necesario». En Didou, S. (Coord.) Internacionalización de la Educación Superior y la Ciencia en América Latina y el Caribe: Un estado del Arte. Caracas: UNESCO-IESALC/Universidad de Panamá.

Drucker, P. (1994). «The Age of Social Transformation» en The Atlantic Monthly, Volume 273, Number 11, Boston.

Fonseca, C. (2001). «Mitos y metas sobre los usos de las nuevas tecnologías en educación» UNESCO- Perspectivas, vol. XXXI, (No. 3) pag.449-464. Recuperado de http://www.unesco.org/new/es/education/resources/onlinematerials/publications/\#toppage [Consulta: 20. 10.2013].

Knight, J. (2010). «Internacionalización de la Educación Superior: Nuevos desarrollos y Consecuencias No Intencionadas», en Boletín IESALC-UNESCO, No. 211.

McLuhan, M. (1986). The Global Village (with Bruce R. Powers) (Oxford University Press).

Moreno, M.M. (2016) La internacionalización del currículo. Estudio de caso comparado. Tesis de maestría en relaciones internacionales. Bogotá: Pontifica Universidad Javeriana, Facultad de Ciencias Políticas y Relaciones Internacionales.

Navarrete, Z. (2010) «La interpelación de una política educativa en la constitución identitaria del pedagogo» en Fuentes, S. y Cruz, O (Coords.) Identidades y políticas educativas, México: Universidad Pedagógica Nacional, pp. 24-37.

Navarrete, Z. y Malaga, S.G. (2014) «Internacionalización de la educación superior. Una mirada desde el Análisis Político de Discurso» en Navarrete, Z. y Navarro, M.A. (Eds.) (2014) Internacionalización y Educación Superior, Estados Unidos de América: Palibrio / Sociedad Mexicana de Educación Comparada, pp. 57-78.

Navarro, M.A. y Navarrete, Z. (2016) «Introducción» en Navarrete, Z. y Navarro, MA.A. (Coords.) Globalización, internacionalización y educación comparada, México: Plaza y Valdés Editores / Sociedad Mexicana de Educación Comparada, pp. 9-19.

Paliwala, A. (2006). «Free Culture, Global Commons and Social Justice» in Information Technology Diffusion. Journal of Information. Law and Technology. 
Robertson, R. (1992). Globalization: Social Theory and Global Culture, Sage Publications, London.

UNESCO (2013). Las tecnologías de la información y la comunicación (TIC) en la educación. Recuperado de http://www. unesco.org/new/es/unesco/themes/icts/ [Consulta: 11.08.2016].

UNESCO (2015). World Education Forum 2015: «Equitable and inclusive quality education and lifelong learning for all by 2030. Transforming lives through education» Recuperado de: http://en.unesco.org/world-education-forum-2015/incheon-declaration (consulta: 15.04.2016)

Valls, J. (2015) La internacionalización de la educación superior, en El Universal (24.08.2016) http://www eluniversal.com. mx/entrada-de-opinion/articulo/jaime-valls-esponda/nacion/2015/11/10/la-internacionalizacion. -de-la.

World Summit on the Information Society (2004). Declaration of Principles. Building the Information Society: a global challenge in the new Millennium. Document WSIS-03/GENEVA/DOC/4-E. Geneva 2003-Tunis 2005.

Zbigniew, B. (1973). La era tecnotronica. Buenos Aires: Paidós. 